Research Article

\title{
Application of 3D Image Technology in Motor and Sensory Nerve Classification
}

\author{
Na Liu \\ College of Human Movement Science, Jilin Sport University, Changchun 130000, China \\ Correspondence should be addressed to Na Liu; 13283723978@hait.edu.cn
}

Received 22 June 2021; Accepted 8 September 2021; Published 28 September 2021

Academic Editor: Gustavo Ramirez

Copyright (C) $2021 \mathrm{Na}$ Liu. This is an open access article distributed under the Creative Commons Attribution License, which permits unrestricted use, distribution, and reproduction in any medium, provided the original work is properly cited.

Objective. To explore the application of 3D image technology in motor and sensory nerve classification. Methods. A total of 200 sections of the $5 \mathrm{~cm}$-long popliteal fossa peroneal nerve from adult volunteers were cut and frozen. The slices were $10 \mathrm{~m}$ thick, and the interval between the slices was $0.25 \mathrm{~mm}$. Acetylcholinesterase histochemical staining was used to observe the changes of nerve bundles under the microscope. The stained sections were transformed into digital images by the digital camera system, and the images were stitched to obtain a two-dimensional panoramic image 100 times magnified. The properties of the functional bundles were manually judged. Using Amira 3.1 three-dimensional reconstruction software to realize the threedimensional reconstruction and visualization of nerve can not only accurately perceive the complex three-dimensional surface structure of nerve, but also arbitrarily display, rotate, scale, and segment the three-dimensional structure inside nerve, and carry out three-dimensional measurement in time. It has made preliminary achievements in brachial plexus, lumbosacral plexus, neural stem functional bundle (group), and intramuscular nerve routing and distribution, including the regeneration process of sensory nerve and three-dimensional reconstruction and visualization of composite tissue containing sensory nerve. Conclusion. Based on histology and computer technology, the functional band of short peroneal nerve can be reconstructed in $3 \mathrm{D}$, which provides a feasible basis for the three-dimensional reconstruction of the functional band of the long peripheral nerve.

\section{Introduction}

Through the study of the internal structure of the peripheral nerve [1], people have gained a deeper understanding of the changes in the type of the fiber bundles in the nerve as well as the qualitative and location of the fiber bundles, which has an important guiding significance for the accurate suture of the nerve bundles with the same functional properties. However, due to the complexity of the internal structure of nerve, the type of nerve bundle changes greatly in a short distance, which makes these research results difficult to be applied in clinic. Both the microanatomical map and histochemical staining sections were isolated sections, lacking continuity and relative positioning marks, which made it difficult to master the memory and could not meet the requirements of the randomized control adjustment of the binding site during the operation [2]. Using computer technology to construct the three-dimensional image of the inner structure of the nerve can make up for the shortcomings mentioned above. At present, there have been studies on the three-dimensional reconstruction of the internal functional bundle of the brachial plexus and the ulnar nerve $[3,4]$, but there are few reports on the three-dimensional reconstruction of the internal functional bundle of the common peroneal nerve. On the basis of the microanatomical and histological studies of the internal structure of the common peroneal nerve, we attempted to reconstruct the three-dimensional image of the path of its functional tract. Computer 3D reconstruction technology was not developed until the end of 1960s. Three-dimensional reconstruction in the early medical field generally uses a microscope projector or tracer to draw the contents of successive sections one by one, then engrave them on a wax plate or cardboard, and finally overlap them to form a three- 
dimensional model. But this three-dimensional reconstruction is time-consuming and imprecise. Due to the development of computer graphics, a series of two-dimensional images can be analyzed, recognized, and processed to establish a three-dimensional image. Due to the development of computer software and hardware, the ability of using computer to reconstruct three-dimensional images of biological specimens has been rapidly developed in the past ten years. At present, 3D image reconstruction using $2 \mathrm{D}$ data sets of biological tissue has been vigorously developed in many fields, including 3D reconstruction of CT and MRI images in medicine, laser confocal reconstruction of biological tissue microstructure, and 3D visual reconstruction of scanning electron microscopy. 3D reconstruction technology has been widely used in the field of medicine and plays an increasingly important role in the development of medicine. In recent years, developed countries have made use of scientific computing visualization technology to visually display the internal structure of the human body. The most typical example is the "Visible Man" project in the United States.

\section{Materials and Methods}

2.1. 3D Reconstruction. The software needed for $3 \mathrm{D}$ reconstruction was also specially developed by the Institute of Computing Technology of Chinese Academy of Sciences with advanced algorithms. After the obtained images were input into the computer, the software was used for 3D reconstruction and postprocessing, and finally, the outer contour of the brachial plexus nerve and the three-dimensional path images of the internal nerve bundles were obtained.

The basic strategy is as follows: 3D body reconstruction between contour lines can be divided into two cases: 3D body reconstruction between convex contour lines and $3 \mathrm{D}$ body reconstruction between nonconvex contour lines. For 3D body reconstruction between convex contour lines, we adopt the synchronous forward method of adjacent contour lines (also known as the correlation model method), which is a heuristic optimization approximation method. The basic idea of this method is that when the triangular surface is used to connect the point rows of two adjacent contours, the connection operation can be carried out as synchronously as possible on the two contours. A better method to reconstruct the triangle between nonconvex contours is to transform the nonconvex contours into convex contours first and then reverse transform them into nonconvex contours after constructing a set of triangular facets between the convex contours.

After the nonconvex contour is converted to convex contour, an acceptable surface between two contours can be constructed by using the synchronous forward method of adjacent contour. The set of triangular surfaces obtained by the above algorithm constitutes the surface of the reconstructed 3D object, which contains the topological and geometric information of the object. At this time, the method of computer graphics can be used to draw. Due to the possible deviation of the original image in the process of acquisition, the reconstructed 3D body has the phenomenon of "sawtooth." Therefore, before rendering, we adopted the "Discrete Energy Method of Smoothing Point of Type Value" for smoothing processing. The program running environment is $\mathrm{VC}++6.0$ \& OpenGL [5].

The software is equipped with a variety of automatic segmentation algorithms, and manual intervention can be carried out to improve the accuracy. According to the program, the contours of all nerve slices can be displayed, so that we can compare the contours of all nerve slices and remove the ones with particularly large offset to improve the accuracy.

2.2. Experimental Subjects. A total popliteal fossa peroneal nerve sample voluntarily donated by a 25 -year-old male patient after hip dissection due to osteosarcoma of the left femur was obtained within 2 hours after the main vessel dissection. The length from the sural nerve branch to the neck of the fibula was about $5 \mathrm{~cm}$, the proximal internal and external width was $2.7 \mathrm{~mm}$, the anteroposterior thickness was $2.2 \mathrm{~mm}$, the distal internal and external width was $3.2 \mathrm{~mm}$, and the anteroposterior thickness was $2.4 \mathrm{~mm}$. The adipose tissue around the epineurium was removed from the specimens, and polymer foam was applied to paste the specimens. The two ends were fixed with pins to prevent the curl and deformation of the nerve and maintain the elongation state. Two hairs (about $0.08 \mathrm{~mm}$ in diameter) were placed on the ventral and posterior sides of the nerve, and a self-made fixator was used to maintain the hair parallel to the longitudinal axis of the nerve to prevent the hair from shrinking or shifting. The hair was embedded with OCT and stored in a refrigerator at $-80^{\circ} \mathrm{C}$. After $28 \mathrm{~h}$, the hair was removed for sectioning and staining.

2.3. Experimental Methods. The nerve specimen was crosssectioned into 10 segments (about $5 \mathrm{~mm}$ in length) with a pathological section knife at $-20^{\circ} \mathrm{C}$, and the OCT was embedded vertically. A total of 200 slices were sectioned using a constant temperature frozen microslicer at $-20^{\circ} \mathrm{C}$ with a thickness of $10 \mathrm{~m}$ and an interval of $0.25 \mathrm{~mm}$ between slices. Within $4 \mathrm{~h}$ after sectioning, acetylcholinesterase histochemical staining was performed by the Karnovsky-Roots $\mathrm{Cu}$ ferrocyanide method. The excess precipitates were washed in PBS at $4^{\circ} \mathrm{C}$ and then dehydrated in a gradient of $95 \%$ alcohol and anhydrous alcohol for $1 \mathrm{~min}$ each time. The xylene was transparent once for $3 \mathrm{~min}$ each time. The pieces were naturally dried and sealed with neutral gum.

2.4. Image Acquisition. After each section was wiped clean with alcohol, photomicrography at 100x magnification was performed using the microimaging system. Photographs of each section were taken in successive sections (9-21 pictures) $(15 \%$ overlap area of $>$ ). AutoStitch image automatic stitching software was used to obtain the slice $2 \mathrm{D}$ panoramic digital image magnified 100 times. The image is saved, each image is $1.5 \mathrm{MB}$ in size, and the total data is $313 \mathrm{MB}$. Image registration and segmentation were performed according to 
the marking points and anatomical signs (such as blood vessels) on the slices by using Photoshop7.0 software. The RGB color mode is transformed into gray-scale mode, so that the two-dimensional image is unified to $720 \times 472 \mathrm{dpi}$, and the image quality of continuous slices is uniform. Then, the automatic contour acquisition function of the software is used to extract the contour information. When the automatic segmentation effect is not good, the drawing board is used for appropriate manual intervention to obtain the satisfactory contour information. If the contour of the nerve bundle changes significantly between two layers due to section injury, contour matching between the upper and lower layers is carried out based on the principle that the size and shape of the corresponding contour between two layers are basically unchanged [6] (Figure 1).

\section{Results and Discussion}

3.1. Changes in the Cross-Sectional Area of Nerve Trunk. The relationship between the cross-sectional area of the nerve trunk and the cross-sectional area of the internal muscle branches of the hand (Table 1): the nerve trunk does not taper from top to bottom due to changes in the content of connective tissue and the number of nerve fibers. However, in the wrist and elbow, the median nerve trunk cross-sectional area increased twice due to the increase of connective tissue proportion. In the deep position of $4 / 16 /$ $13 / 16$ segment and the upper arm above $2 / 8$ segment, the nerve trunk became thinner due to the decrease of connective tissue content and the relative concentration of nerve fibers. Investigating its reason, it should be with the elbow and wrist nerve to be pulled and the friction opportunity is more related.

Changes of median nerve cross-sectional area (Figure 2): there were two regions of median nerve trunk with significantly larger cross-sectional area between the dividing plane of thenar muscular branches (1/16 plane) and between the $12 / 16$ plane and the 218 plane. Among them, the median nerve trunk had the largest cross-sectional area at the plane of interthelar muscular branches and the plane of interthelar muscular branches, which were, respectively, at the interthelar skeletal line plane. Microscopic observation showed obvious hyperplasia of connective tissue in the two regions [7].

As can be seen from the results in Table 2, the intermuscular branch of the median nerve occupies a small proportion in the whole nerve trunk, with the original size (0/16 plane) of $4 \%$, and mostly crosses with other sensory tract branches in a short distance, with a short separation range.

3/16 plane far: median nerve thenar muscle branch can be separate, and connective tissue content is higher in the nerve stem at this stage; the bundle branch on the nerve trunk under representation of relative function is particularly important, but the median nerve in the period of nerve damage should determine the distance of the thenar muscle branch section (in the nerve trunk bending side or scratching hand side) and then stitching line "membrane" beam. Since other bundle groups are all sensory bundle groups and can be roughly divided into three relatively concentrated bundle groups, it is also better to use "bundle group" to stitch $\mathrm{Z}$ from $\mathrm{O}$.

Before the fusion of the proximal and interosseous volar nerves into the nerve trunk at the 3/16 plane, the thenar muscle branches have interwoven into the nerve trunk, and the connective tissue content in this segment of the nerve trunk has been significantly reduced. When this segment of the nerve is injured, the epineurium can be sutured under the condition that the posterior direction of the nerve trunk is in alignment with the priority. During the nerve anatomy of this segment, it should be noted that the distal branches of flexor digitalis superficial branch all merged into the nerve trunk from the ulnar side of the nerve trunk except the palmar cutaneous branch near the 4/16 plane, suggesting that the nerve trunk should be disintegrated on the lateral side of the nerve trunk during surgery to reduce the nerve bundle branch injury.

Volar nerve between bone into the nerve trunk: after nerve injury repair, full attention should be given to the bone between the volar nerve as a bundle of the motor group, often in the nerve trunk back or foot back, and the nerve trunk is mainly composed of beam from the hand, so it should first ensure the involution of volar nerve between bones, uneffective "beam membrane" suture, and nerve trunk lines membrane suture.

3.2. Image Registration and Segmentation. By using Photoshop7.0 software, image registration was carried out according to the marking points and anatomical signs (such as blood vessels) on the sections. The RGB color mode is transformed into gray mode [8], so that the twodimensional image is unified to $720 \times 472 \mathrm{dpi}$, and the image quality of continuous slices is homogenized. Then, the automatic contour acquisition function of the software is used to extract the contour information. When the automatic segmentation effect is not good, the drawing board is used for appropriate manual intervention to obtain the satisfactory contour information. If the contour of the nerve bundle changes significantly between two layers due to section injury, the contour matching process between the upper and lower layers is carried out based on the principle that the size and shape of the corresponding contour between two layers are basically unchanged. According to the results of microanatomy and AChE staining, the functional properties of the nerve bundles after registration and segmentation were classified. The deep peroneal nerve is located in the anterolateral part of the nerve trunk, and the superficial peroneal nerve is located in the medial part of the nerve trunk. Because it contains a large number of sympathetic nerves, the positive distribution area of crumb nerve bundle becomes sensory nerve bundle, and it is mainly evenly distributed positive motor nerve bundle, which is mainly composed of motor nerve fiber bundle or mixed nerve bundle. Different data channels are built for different nerve bundles so that they can be displayed independently in $3 \mathrm{D}$ reconstruction. 
TABLE 1: Size changes of median nerve trunk in anteroposterior, left and right meridians, and cross-sectional area.

\begin{tabular}{|c|c|c|c|}
\hline & About size $(\mathrm{mm})$ & Diameter before and after $(\mathrm{mm})$ & Cross-sectional area $\left(\mathrm{mm}^{2}\right)$ \\
\hline Tell flat & $5.24 \pm 1.35$ & $2.82 \pm 0.97$ & $8.41 \pm 2.25$ \\
\hline $0 / 16$ plane & $4.57 \pm 1.20$ & $2.61 \pm 0.61$ & $7.10 \pm 2.63$ \\
\hline $1 / 16$ plane & $3.64 \pm 1.04$ & $2.89 \pm 0.68$ & $6.28 \pm 2.73$ \\
\hline $2 / 16$ plane & $3.20 \pm 0.92$ & $3.11 \pm 0.79$ & $5.80 \pm 1.85$ \\
\hline $3 / 16$ plane & $3.42 \pm 0.82$ & $3.02 \pm 0.84$ & $6.09 \pm 1.98$ \\
\hline $4 / 16$ plane & $3.22 \pm 0.45$ & $2.74 \pm 0.75$ & $5.26 \pm 1.53$ \\
\hline $5 / 16$ plane & $3.17 \pm 0.52$ & $2.65 \pm 0.51$ & $5.13 \pm 1.67$ \\
\hline $6 / 16$ plane & $3.01 \pm 0.43$ & $2.74 \pm 0.44$ & $4.93 \pm 1.05$ \\
\hline $7 / 16$ plane & $3.07 \pm 0.28$ & $2.64 \pm 0.67$ & $4.91 \pm 1.60$ \\
\hline $8 / 16$ plane & $2.91 \pm 0.57$ & $2.68 \pm 0.55$ & $4.70 \pm 1.46$ \\
\hline 9/16 plane & $2.89 \pm 0.42$ & $2.52 \pm 0.55$ & $4.33 \pm 0.90$ \\
\hline $10 / 16$ plane & $2.93 \pm 0.52$ & $2.60 \pm 0.58$ & $4.50 \pm 0.95$ \\
\hline $11 / 16$ plane & $2.94 \pm 0.50$ & $2.74 \pm 0.62$ & $4.87 \pm 1.48$ \\
\hline $12 / 16$ plane & $2.72 \pm 0.56$ & $3.03 \pm 0.81$ & $4.90 \pm 1.44$ \\
\hline $13 / 16$ plane & $2.88 \pm 0.65$ & $3.75 \pm 1.16$ & $6.28 \pm 1.82$ \\
\hline $14 / 16$ plane & $2.95 \pm 1.28$ & $4.15 \pm 1.04$ & $6.99 \pm 2.22$ \\
\hline $15 / 16$ plane & $3.15 \pm 1.52$ & $4.57 \pm 1.65$ & $7.76 \pm 2.42$ \\
\hline $16 / 16$ plane & $3.40 \pm 1.21$ & $4.15 \pm 0.81$ & $8.24 \pm 2.69$ \\
\hline $1 / 8$ plane & $3.07 \pm 1.01$ & $3.77 \pm 0.99$ & $6.67 \pm 1.85$ \\
\hline $2 / 8$ plane & $2.97 \pm 0.82$ & $3.47 \pm 0.78$ & $6.05 \pm 1.7$ \\
\hline $3 / 8$ plane & $2.90 \pm 0.64$ & $3.48 \pm 0.66$ & $6.03 \pm 1.71$ \\
\hline 4/8 plane & $2.81 \pm 0.52$ & $3.29 \pm 0.70$ & $5.58 \pm 1.79$ \\
\hline $5 / 8$ plane & $2.75 \pm 0.66$ & $3.47 \pm 0.85$ & $5.68 \pm 2.00$ \\
\hline $6 / 8$ plane & $2.86 \pm 0.81$ & $3.5 \pm 0.69$ & $5.93 \pm 1.66$ \\
\hline $7 / 8$ plane & $2.88 \pm 0.88$ & $3.47 \pm 0.78$ & $6.04 \pm 2.66$ \\
\hline $8 / 8$ plane & $2.93 \pm 0.83$ & $3.26 \pm 0.64$ & $5.68 \pm 1.77$ \\
\hline
\end{tabular}

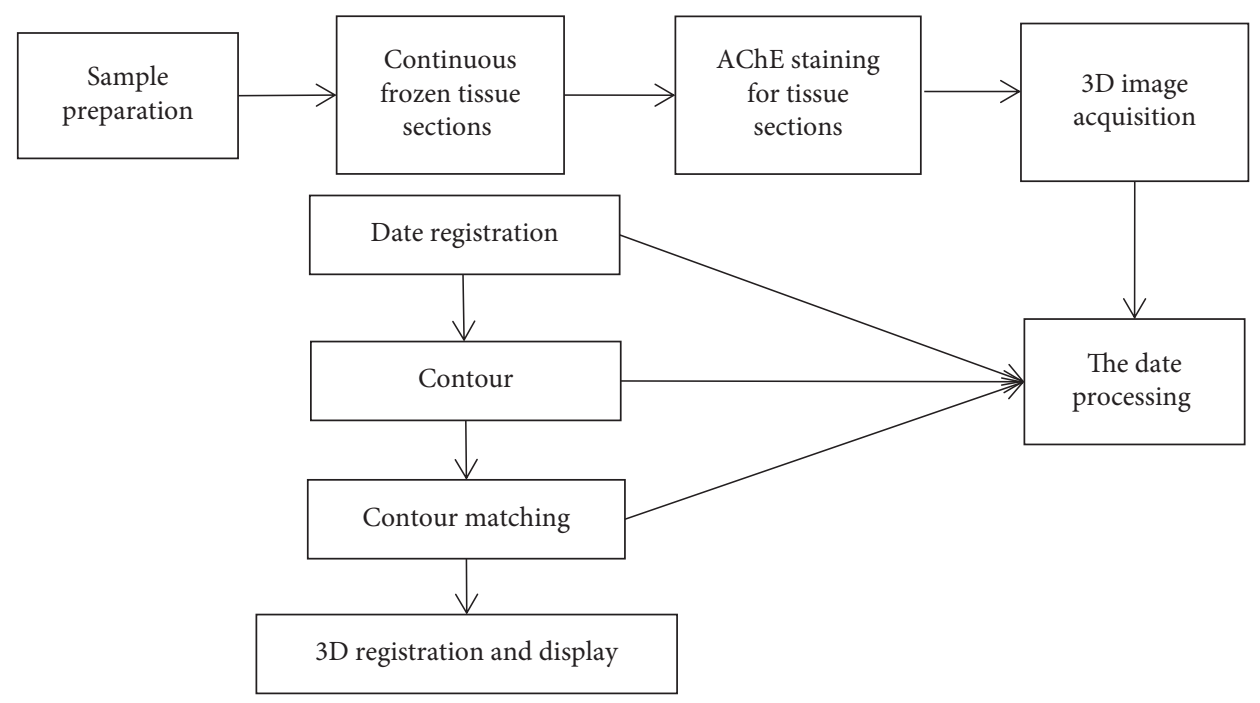

Figure 1: Computerized 3D reconstruction of brachial plexus.

3.3. Two-Dimensional Image Observation of Continuous Cross Section of Common Peroneal Nerve. This segment of the common peroneal nerve is located behind the small head of the fibula and belongs to the distal end of the common peroneal nerve. The lateral head of the sural nerve from which the common peroneal nerve divides has already divided at its proximal end, which is closer to the target organ, especially the superficial peroneal nerve. Proximal crosssectional images [9] showed that the superficial peroneal nerve and the deep peroneal nerve were divided into distinct regions, among which the superficial peroneal nerve was located in the intracranial posterior and had a total of 4 bundles, including 2 sensory nerve bundles and 2 mixed nerve bundles dominated by motor nerve fibers. The deep peroneal nerve is located on the anterior and external side of the nerve. There are 4 bundles, including 3 large mixed bundles and 1 small motor bundle. After walking $2.5 \mathrm{~cm}$, the common peroneal nerve was observed in cross section. The sensory tracts of the superficial peroneal nerve formed 4 bundles from 2 bundles. The deep peroneal nerve is divided 


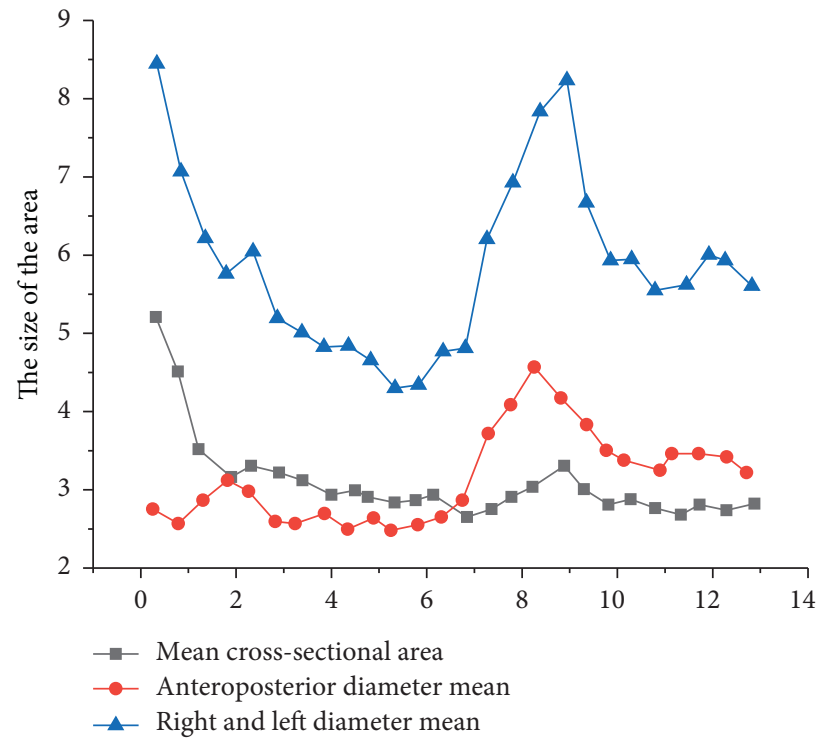

Figure 2: Changes of anterior and posterior meridian area of the median nerve.

TABLe 2: The size and proportion of the cross-sectional area between median nerve and thenar fascicular branch.

\begin{tabular}{lccc}
\hline The plane & Cross-sectional area of thenar muscle branch $(\mathrm{A})$ & Nerve trunk $(A)$ area & Area ratio $(\mathrm{a} / \mathrm{A})$ \\
\hline Tell flat & $0.36 \pm 0.11$ & 8.41 & 0.04 \\
0/16 plane & $0.25 \pm 0.08$ & 6.1 & 0.28 \\
1/16 plane & $0.33 \pm 0.28$ & 5.80 & 0.05 \\
2/16 plane & $0.81 \pm 0.62$ & 6.09 & 0.14 \\
3/16 plane & $1.49 \pm 0.63$ & 5.26 & 0.24 \\
4/16 plane & $2.09 \pm 0.93$ & 5.13 & 0.40 \\
5/16 plane & $3.29 \pm 0.36$ & 0.64 \\
\hline
\end{tabular}

into 6 mixed nerve bundles. Further down $2.5 \mathrm{~cm}$, the superficial peroneal nerve formed 7 bundles, 3 of which were sensory nerve bundles and 4 of which were mixed nerve bundles mainly composed of motor nerve fibers. The mixed nerve bundles mainly composed of motor nerve fibers in this segment of the superficial peroneal nerve only changed in position within the range of $2.3 \mathrm{~cm}$, without any bundle crossover and kept to the rear of the fibular neck. There are 6 bundles of deep peroneal nerve, all of which are mixed nerves, and there are a lot of nerve-crossing changes between the 6 bundles during the course.

3.4. Image Registration and Segmentation. Image registration and segmentation are the difficulties and key points in $3 \mathrm{D}$ reconstruction [10]. In the process of slicing, dyeing, microphotography, and image mosaic, the image may produce changes such as displacement, rotation, and shrinkage. Therefore, it is necessary to preprocess the original 2D digital image before $3 \mathrm{D}$ reconstruction to obtain the volume data that can be used for 3D data visualization, including image registration, image segmentation, and contour matching. Existing studies of nerve internal microdissection threedimensional reconstruction all need to develop some advanced computer calculation method to automatically finish this job, but in the process of slicing, it may produce tissue section of curl, such as tensile and compression. And the change of a single slice may lead to fracture and crease, and the tissue may shrink after fixation and dehydration. Therefore, there is no suitable method to achieve automatic and accurate contour acquisition and matching of neural cross section at present, which usually requires more manual intervention. We use Photoshop7.0 software developed a slice image registration process [11], and based on the correspondence between the two layers of slice contour shape, the basic rule of constant size up and down between the two layers of image registration and contour matching processing, the satisfactory effect of $3 \mathrm{~d}$ reconstruction is obtained, but the disadvantage is that it needs to be more accurate in the case of manual operation and it has a lot of work.

\section{Conclusions}

Two-dimensional images of tissue sections were obtained by using the optical microscope. Due to a series of difficulties in positioning, image distortion, and image segmentation, 3D reconstruction technology still lags behind the development of CT, MRI, and 3D laser scanning confocal microscope. Therefore, the three-dimensional reconstruction of the internal microanatomy of the peripheral nerve is still in the preliminary exploration stage. However, with the deepening 
of research, the three-dimensional reconstruction technology of peripheral nerve will be improved. Through the threedimensional reconstruction of peripheral nerve function, a relatively complete and accurate virtual database is established, and the matching scheme of peripheral nerve injury repair and nerve transplantation is reasonably selected. It is of great value for the diagnosis and accurate positioning of various nerve injuries and diseases and the intuitive teaching of nerve microanatomy. It will also provide a research platform and technical support for higher level teaching, scientific research, and clinical application in the field of peripheral nerves.

\section{Data Availability}

The data used to support the findings of this study are available from the corresponding author upon request.

\section{Conflicts of Interest}

The author declares that there are no conflicts of interest.

\section{References}

[1] M. Z. Baig and M. Kavakli, "Classification of user competency levels using EEG and convolutional neural network in 3D modelling application," Expert Systems with Applications, vol. 146, Article ID 113202, 2020.

[2] L. Malinovsk, "Sensory nerve formations in the skin and their classification," Microscopy Research and Technique, vol. 34, no. 4, pp. 283-301, 2015.

[3] C. Lastoria, S. Bido, P. Ceriana, and M. Vitacca, "Implementation of the icd-icf model in rehabilitation medicine: report of a clinical case in respiratory rehabilitation," Giornale Italiano di Medicina del Lavoro ed Ergonomia, vol. 41, no. 2, pp. 150-155, 2019.

[4] G. Galardi, S. Amadio, L. Maderna et al., "Electrophysiologic studies in tarsal tunnel syndrome. Diagnostic reliability of motor distal latency, mixed nerve and sensory nerve conduction studies," American Journal of Physical Medicine \& Rehabilitation, vol. 73, no. 3, pp. 193-198, 2016.

[5] B. Federico, G. G. Omar, A. Enol et al., "Functional and morphologic alterations in mechanical, polymodal, and cold sensory nerve fibers of the cornea following photorefractive keratectomy," Investigative Ophthalmology \& Visual Science, vol. 59, no. 6, pp. 2281-2292, 2018.

[6] E. Verdú, J. J. Vilches, F. J. Rodríguez, D. Ceballos, A. Valero, and X. Navarro, "Physiological and immunohistochemical characterization of cisplatin-induced neuropathy in mice," Muscle \& Nerve, vol. 22, no. 3, pp. 329-340, 2015.

[7] P. Dubovy and H. Aldskogius, "Degeneration and regeneration of cutaneous sensory nerve formations," Microscopy Research and Technique, vol. 34, no. 4, pp. 362-375, 2015.

[8] A. I. Portugués, I. Alcalde, O. González, J. Gallar, C. Belmonte, and J. Merayo-Lloves, "Decreased basal tear in aged mice is linked to morphological and functional changes in corneal sensory nerve fibers," Acta Ophthalmologica, vol. 92, no. s253, 2015.

[9] S. J. Oh, S. Hemmi, and Y. Hatanaka, "Diagnostic markers of axonal degeneration and demyelination in sensory nerve conduction," Muscle \& Nerve, vol. 53, no. 6, pp. 866-871, 2016.
[10] S. Sarram, K. F. Lee, and M. R. Byers, "Dental innervation and CGRP in adult p75-deficient mice," Journal of Comparative Neurology, vol. 385, no. 2, pp. 297-308, 2015.

[11] F. Gonzalez-Perez, A. Alé, D. Santos et al., "Substratum preferences of motor and sensory neurons in postnatal and adult rats," European Journal of Neuroscience, vol. 43, no. 3, pp. 431-442, 2016 